\title{
Three-body resonances by complex scaling
}

\author{
Attila Csótó* \\ W. K. Kellogg Radiation Laboratory, 106-38, California Institute of Technology, \\ Pasadena, California 91125, USA
}

(June 3, 2022)

\begin{abstract}
It is demonstrated that the complex scaling method can be used in practical calculations to localize three-body resonances. Our model example emphasizes the fact that in three-body systems several essentially different asymptotic behaviors can appear. We show that the possibility of these different asymptotic configurations can lead to an apparent, resonance like structure in the three-body continuum.
\end{abstract}

PACS numbers: 21.45.+v, 24.30.Gd 
Three-body resonances, systems which decay into three-body final states, play important role in few-body physics. The description of these states are somewhat easier than that of the scattering processes which lead three (or more) particles in the outgoing channel. The study of three-body resonances hopefully will help us to clarify some points of the still not totally solved scattering problem.

One of the main difficulties in the general formulation of the quantum mechanical N-body resonance (and scattering) problem is the prescription of the asymptotic behavior. There is fundamental difference between the problems where only short range interactions occur and those where a long range force, e.g. the Coulomb force, is present [1]. There are methods which eliminate the explicit reference to the unknown or partially known asymptotics, e.g. the J-matrix method [2], and the potential separable expansion method [3], but up till now they have been developed only for two-body systems. In the case of bound states the question of the asymptotic behavior is not a serious problem. The majority of the methods work because in most cases the bound state asymptotics hardly affects the determination of physically observable quantities. However, in the case of resonances and scattering states, the asymptotic behavior plays a crucial role.

The complex scaling method (CSM) [4] reduces the description of resonant states to the description of bound states, thus avoids the problem of asymptotics. This method handles the non-Coulomb and the Coulomb cases on equal footing. There is an extension of this method to two-body scattering states, too [5]. It is an intriguing question whether this method can be extended to the general many-body scattering states. Up till now, three-body resonances have been investigated e.g. using the Faddeev method [6], by real stabilization [7], and in the time delay matrix formalism [8]. In this paper we study them using the complex scaling method. Although the CSM was used to describe three-body systems above the three-body breakup [9], the authors did not specify how they identified three-body resonances. This research was prompted by the contradicting theoretical results concerning the existence of a soft dipole resonance in the neutron halo nucleus ${ }^{6} \mathrm{He}$ [10, [1]. What makes this problem difficult, is the mixture of the underlying nuclear physics and three-body dynamics. Here we want to clarify some points of the three-body dynamics.

For the sake of simplicity, we recall the main points of the CSM in two-body context. In the coordinate space, resonance eigenfunctions, corresponding to the complex energy solutions of the

$$
\widehat{H}|\Psi\rangle=(\widehat{T}+\widehat{V})|\Psi\rangle=E|\Psi\rangle
$$

Schrödinger equation, show oscillatory behavior in the asymptotic region, with exponentially growing amplitude, $\sim \exp [i(\kappa-i \gamma) r](\kappa, \gamma>0)$. Thus, they are not elements of the $L^{2}$ space. The complex scaling method means that instead of Eq. (1), we solve the eigenvalue problem of the transformed Hamiltonian $\widehat{H}_{\theta}=\widehat{U}(\theta) \widehat{H} \widehat{U}^{-1}(\theta)$ :

$$
\widehat{H}_{\theta}\left|\Psi_{\theta}\right\rangle=E_{\theta}\left|\Psi_{\theta}\right\rangle .
$$

$\widehat{U}(\theta)$ is an unbounded similarity transformation [12], which, in the coordinate space, acts on a function $f(r)$ such that

$$
\widehat{U}(\theta) f(r)=e^{3 i \theta / 2} f\left(r e^{i \theta}\right) .
$$


(If $\theta$ is real, $\widehat{U}(\theta)$ means a rotation into the complex coordinate plane, if it is complex, it means a rotation and scaling.) The two problems are connected by the Aguilar-BalslevCombes theorem [13]: If $\widehat{V}$ is a (dilation) analytic operator, then (i) the bound eigenstates of $\widehat{H}$ are the eigenstates of $\widehat{H}_{\theta}$, regardless of the actual value of $\theta$, within $0 \leq \theta<\pi / 2$; (ii) the continuous spectrum of $\widehat{H}$ will be rotated by an angle $2 \theta$; (iii) a complex generalized eigenvalue of Eq. (2) $, E_{\mathrm{res}}=\varepsilon-i \frac{1}{2} \Gamma, \varepsilon, \Gamma>0$ (with the wave number $k_{\mathrm{res}}=\kappa-i \gamma, \kappa, \gamma>0$ ), belongs to the proper spectrum of $\widehat{H}_{\theta}$ provided $2 \theta>\left|\arg E_{\text {res }}\right|$. Roughly speaking, the complex scaling transformation changes the asymptotic wave function from $\exp [i(\kappa-i \gamma) r]$ to $\exp [i(\kappa-i \gamma) r \exp (i \theta)]$, which, in the case of $2 \theta>\left|\arg E_{\text {res }}\right|=2\left|\arg k_{\text {res }}\right|$, makes the diverging wave function localized.

If we have $N$ particles, we can transform the problem from one-particle coordinates to certain interparticle relative coordinates (Jacobi coordinates):

$$
\left\{\mathbf{r}_{1}, \mathbf{r}_{2}, \ldots, \mathbf{r}_{N}\right\} \rightarrow\left\{\mathbf{t}_{1}, \mathbf{t}_{2}, \ldots, \mathbf{t}_{N-1}\right\}
$$

where the origin is fixed at the center of mass, so that $\sum_{i=1}^{N} m_{i} \mathbf{r}_{i}=0$, where $m_{i}$ are the particle masses. The application of the CSM in this case means that we transform all relative coordinates under the action of $\widehat{U}(\theta)$. Note that the (4) transformation is linear, therefore if we carry out the complex scaling transformation in a certain set of Jacobi coordinates, it results in the same transformation in all other possible sets of relative coordinates. It means that we cannot choose the rotation angles independently, like in the multichannel CSM |14], in the different configurations.

Our present model problem consists of three particles with masses $m_{1}, m_{2}$, and $m_{3}$. We choose $m_{1}=m_{2}$ so as to have only two different systems of Jacobi coordinates. The interactions between the particles are separable forces

$$
\widehat{V}_{i j}=\left|\varphi_{0}(b)\right\rangle \lambda_{i j}\left\langle\varphi_{0}(b)\right|, \quad j>i=1,2,3
$$

where $\left|\varphi_{0}(b)\right\rangle$ is the eigenfunction of the three dimensional harmonic oscillator with $n=l=$ $0, b$ is the oscillator size parameter, and $\lambda_{i j}$ are the potential strengths. In coordinate space the interactions depend on the various relative coordinates. Each interaction has a natural Jacobi coordinate system in which it has the simplest form, e.g. in the (12)3 coordinate system (there is a relative coordinate between particles 1 and $2, \mathbf{t}_{12}$ and another between $(1,2)$ and $\left.3, \mathbf{t}_{(12) 3}\right)$ the $V_{12}$ interaction depends only on $\mathbf{t}_{12}$.

The kinetic energy operator is easy to express in any coordinate system, e.g. in (12)3 it looks like

$$
T=-\frac{\hbar^{2}}{2}\left[\frac{1}{\mu_{12}} \boldsymbol{\Delta}_{\mathbf{t}_{12}}+\frac{1}{\mu_{(12) 3}} \boldsymbol{\Delta}_{\mathbf{t}_{(12) 3}}\right],
$$

where the Laplace operators are differential operators in the appropriate Jacobians. It is easy to show that the application of the (3) complex scaling transformation to our potential and kinetic energy operators is equivalent with the change of $b$ to $b \exp (i \theta)$ in (5), and the multiplication of the right-hand side of (6) by $\exp (-2 i \theta)$, respectively.

As the CSM localizes the resonant wave function, we can use any bound-state method to describe them. Here we use the wave function expansion method. We consider three different wave functions. In coordinate space 


$$
\begin{aligned}
& \Psi_{1}=\sum_{i j} c_{i j} \varphi_{i}\left(\mathbf{t}_{12}\right) \varphi_{j}\left(\mathbf{t}_{(12) 3}\right) \\
& \Psi_{2}=\sum_{i j} d_{i j} \varphi_{i}\left(\mathbf{t}_{23}\right) \varphi_{j}\left(\mathbf{t}_{(23) 1}\right)
\end{aligned}
$$

and the unification of the above two, $\Psi_{3}=\Psi_{1}+\Psi_{2}$. The expansion coefficients $c$ and $d$ are to be determined from a variational principle. Our potentials act only between $s$-waves, therefore each oscillator function carries zero angular momentum in (7) and (8). We choose the oscillator size parameter of the wave functions, $\bar{b}$, different from $b$ in order to make the trial functions more flexible. The summation limits in the wave functions are chosen to reach stable convergence.

For the necessary matrix elements we need to calculate the overlap of the product oscillator states between different Jacobi coordinate systems, and the Laplace operator between such states. As examples, we show typical terms that appear in the overlap, kinetic energy, and potential matrix elements:

$$
\begin{gathered}
\left\langle\varphi_{i}\left(\mathbf{t}_{23}\right) \varphi_{j}\left(\mathbf{t}_{(23) 1}\right) \mid \varphi_{k}\left(\mathbf{t}_{12}\right) \varphi_{l}\left(\mathbf{t}_{(12) 3}\right)\right\rangle, \\
\left\langle\varphi_{i}\left(\mathbf{t}_{23}\right) \varphi_{j}\left(\mathbf{t}_{(23) 1}\right)\left|\Delta_{\mathbf{t}_{12}}\right| \varphi_{k}\left(\mathbf{t}_{12}\right) \varphi_{l}\left(\mathbf{t}_{(12) 3}\right)\right\rangle,
\end{gathered}
$$

and

$$
\left\langle\varphi_{i}\left(\mathbf{t}_{23}\right) \varphi_{j}\left(\mathbf{t}_{(23) 1}\right) \mid \varphi_{0}\left(\mathbf{t}_{12}\right)\right\rangle\left\langle\varphi_{0}\left(\mathbf{t}_{12}\right) \mid \varphi_{k}\left(\mathbf{t}_{12}\right) \varphi_{l}\left(\mathbf{t}_{(12) 3}\right)\right\rangle \text {. }
$$

Using the Talmi-Moshinsky-Tobocman transformation [15 we can express a product of oscillator states, given in a certain Jacobi coordinate system $(\alpha)$, in terms of product oscillator states in another system $\left(\alpha^{\prime}\right)$, e.g.

$$
\varphi_{i}^{\alpha}\left(\mathbf{t}_{23}\right) \varphi_{j}^{\alpha}\left(\mathbf{t}_{(23) 1}\right)=\sum_{k, l} a_{i j k l}^{\alpha \alpha^{\prime}} \varphi_{k}^{\alpha^{\prime}}\left(\mathbf{t}_{12}\right) \varphi_{l}^{\alpha^{\prime}}\left(\mathbf{t}_{(12) 3}\right)
$$

where the sum is finite, and the transformation coefficients can be calculated, e.g. by the 16 program. Using these transformations, and in addition the overlap between two oscillator functions with different size parameters, and the matrix element of the Laplace operators between such oscillator functions (for the formulae see e.g. [17]), all necessary matrix elements can be calculated analytically.

We choose $m_{1}=m_{2}=2$, and $m_{3}=4$ ( $\hbar=1$ and atomic mass units are used), $b=1.0$, and $\bar{b}=2.0$. The use of separable interactions allows us to set up their strengths in such a way that resonances occur at prescribed energies in the two-body subsystems [18]. The choice $\lambda_{12}=0.6377+i 0.0697$ results in a resonance in the $(1,2)$ subsystem at $E=1.5-i 0.5$. The $\lambda_{13}=\lambda_{23}=1.0$ strengths give a resonance in the $(1,3)$ and $(2,3)$ subsystems at $1.7553-i 0.2438$ energy. As an illustrative example we show in Fig. 11(a) the result of a CSM calculation for the $(1,2)$ subsystem. The working mechanism of the method is clearly seen, the discretized continuum points are rotated, and the resonance is revealed.

In Figs. 1(b)-(d) we show the results of the three-body CSM calculations using the $\Psi_{1}$, $\Psi_{2}$, and $\Psi_{3}$ trial functions, respectively. We can see that as the rotation angles are large 
enough to localize the resonances in the subsystems, there are discretized continuum points lying on strait half-lines which start from the position of the resonances of the subsystems. These starting points act as non real thresholds. For example, in Fig. 1)(b) the half-line starts at $1.5-i 0.5$ which is the resonance energy in the (12) subsystem. This is in full agreement with the mathematical theorems [19,20]. In addition to these continuum points, we can see that there is an isolated point at $4.128-i 0.337$ in each figure. We can identify this point as a three-body resonance. The fact that this point occur in each figure shows that this state of the three-body system can show up both 3(12) and 1(23) asymptotic behaviors. This is exactly what we expect from a three-body resonance. This behavior gives us a method to identify three-body resonances in practical calculations. But what can we say about the continuum states which lie on the half-lines starting from the resonance energies of the subsystems? In these states e.g. in the 1(23) configuration there is a resonant state in the $(2,3)$ system and a scattering state between 1 and $(2,3)$. These continuum states are essentially different from a pure three-body scattering state which is represented by the continuum points lying on the half-lines start at the origin. The resonance+scattering type continuum states can represent some kind of sequential decay, where the life time of a quasistationary subsystem, $(2,3)$, is longer than the time needed for 1 and $(2,3)$ to be scattered off.

The fact that different type of continuum states can be present in a three-body system means that the three-body continuum has structure in addition to the three-body resonances. Let us speculate a bit about this additional structure. In Fig. 2 we show the distribution of the continuum points (in 0.2 wide energy bins) of the model whose wave function is $\Psi_{3}$. We note here that there is no qualitative difference between not complex scaled $(\theta=0)$ and complex scaled results because the CSM causes only a contraction of the continuum points, while the resonances remain stable. We can see in Fig. 2 the resonant structure around 4.2. But, in addition, we see structures, the concentration of the continuum points, around 1.6 and 2.0 which values coincide the real parts of the resonance energies of the subsystems. These resonant-like structures are apparent and they are the consequence of the fact that if the energy is larger than the threshold energy of a subsystem's resonance, a new, resonance+scattering, asymptotic behavior can appear. We should note that the shape of the background distribution in Fig. 2 is surprising. In the case of two particles, the wave function expansion method can be considered as if we closed our system into a box whose size is the finite spatial region of the trial function. This leads to a $E_{n} \sim n^{2}(n=1,2, \ldots)$ spectrum. From this, it follows that the number of continuum points which are in a $\Delta E$ interval around $E$ (if $\Delta E$ is small) is $\Delta N \sim 1 / \sqrt{E} \Delta E$. We checked in a two-body model that this is a really good approximation. Our three-body spectrum in Fig. 2 is, however, strongly differs from such a shape. In the case of three particles, the Schrödinger equation can be cast, in the hyperspherical coordinate, into a form which is similar to a two-body equation, see e.g. Ref. 21]. If all angular momenta are zero, in this reformulated Schrödinger equation a centrifugal barrier occures with $L=3 / 2$. This nonzero $L$ really leads to a reduction of the low energy solutions, but this is a marginal decrease. There must be another effect which supresses the low energy spectrum.

In conclusion, we showed that the complex scaling method can be used in practical three-body calculations. In this method, three-body resonances can be identified by those resonant energy solutions which appear in all Jacobi coordinate system. We pointed out that 
the possibility of resonant+scattering type asymptotic behaviors can lead to an apparent structure in the three-body continuum. The case of the ${ }^{6} \mathrm{He}$ soft dipole mode is very similar to our present example. That nucleus is a genuine three-body, $\alpha+n+n$, system [22, 23]. There are two resonances in the $\alpha+n$ subsystem at $0.89-i 0.60 \mathrm{MeV}$ and $4-i 4 \mathrm{MeV}$ energies [24]. (And there is an antibound state in the $n+n$ subsystem, which is out of the scope of this article.) From what we can learn our present model, it is fairly possible that the structure in the ${ }^{6} \mathrm{He}$ continuum, which is interpreted as a fingerprint of a three-body resonance, the so-called soft dipole resonance, is nothing but a consequence of the three-body dynamics. Of course here we considered only one side of the problem. The question of the ${ }^{6} \mathrm{He}$ soft dipole resonance can only be answered in a model which contains both the nuclear physics and the three-body dynamics properly. The application of the complex scaling method in a realistic model of ${ }^{6} \mathrm{He}$ is in progress.

This work was supported by a Fulbright Fellowship (USA), a Research Fellowship from the Science Policy Office (Belgium) and by OTKA grant No. 3010 (Hungary). I wish to thank Professor D. Baye, Professor B. Gyarmati and Dr. Z. Papp for useful discussions. 


\section{REFERENCES}

* E-mail address: H988CSO@HUELLA.BITNET

On leave from: Institute of Nuclear Research of the Hungarian Academy of Sciences, P.O.Box 51, Debrecen, H-4001, Hungary

[1] R. G. Newton, Scattering theory of waves and particles, (Springer-Verlag, New York, 1982).

[2] H. A. Yamani and L. Fishman, J. Math. Phys. 16, 410 (1975).

[3] B. Gyarmati and A. T. Kruppa, Phys. Rev. C 34, 95 (1986); Z. Papp, J. Phys A 20, 153 (1987); Phys. Rev. C 38, 2457 (1988); and private communication.

[4] Y. K. Ho, Phys. Rep. 99, 1 (1983); N. Moiseyev, P. R. Certain, and F. Weinhold, Mol. Phys. 36, 1613 (1978); Proceedings of the Sanibel Workshop Complex Scaling, 1978 [Int. J. Quantum Chem. 14, 343 (1978)]; B. R. Junker, Adv. At. Mol. Phys. 18, 207 (1982); W. P. Reinhardt, Annu. Rev. Phys. Chem. 33, 223 (1982); ResonancesThe Unifying Route Towards the Formulation of Dynamical Processes, Foundations and Applications in Nuclear, Atomic and Molecular Physics, edited by E. Brändas and N. Elander, Lecture Notes in Physics Vol. 325 (Springer-Verlag, Berlin, 1989).

[5] T. N. Resigno and P. Reinhardt, Phys. Rev. A 8, 2828 (1973).

[6] Y. Matsui Phys. Rev C 22, 2591 (1980); A. Eskandarian and I. R. Afnan, Phys. Rev. C 46, 2344 (1992).

[7] P. Froelich, K. Szalewicz, and R. Stab, Phys. Lett. A 129, 321 (1988).

[8] J. P. Svenne, T. A. Osborn, G. Pisent, and D. Eyre, Phys. Rev. C 40, 1136 (1989).

[9] C-Y. Hu and A. K. Bhatia, Phys Rev. A 42, 5769 (1990); P. Froelich, A. Flores-Riveros, and S. A. Alexander, Phys. Rev. A 46, 2330 (1992).

[10] Y. Suzuki, Nucl. Phys. A528, 395 (1991).

[11] B. V. Danilin, M. V. Zhukov, J. S. Vaagen, and J. M. Bang, Phys. Lett. B 302, 129 (1993); L. S. Ferreira, E. Maglione, J. M. Bang, I. J. Thompson, B. V. Danilin, M. V. Zhukov, and J. S. Vaagen, Phys. Lett. B 316, 23 (1993).

[12] P. O. Löwdin, Adv. Quantum Chem. 19, 87 (1988).

[13] J. Aguilar and J. M. Combes, Commun. Math. Phys. 22, 269 (1971); E. Balslev and J. M. Combes, ibid. 22, 280 (1971); B. Simon, ibid. 27, 1 (1972).

[14] A. Csótó, Phys. Rev. A 48, 3390 (1993).

[15] W. Tobocman, Nucl. Phys. A357, 293 (1981).

[16] Y. Gan, M. Gong, C. Wu, and C. Bao, Comp. Phys. Commun. 34, 387 (1985).

[17] A. T. Kruppa and K. Katō, Prog. Theor. Phys. 84, 1145 (1990).

[18] A. Csótó, B. Gyarmati, A. T. Kruppa, K. F. Pál, and N. Moiseyev, Phys. Rev. A 41, 3469 (1990).

[19] E. Balslev, in Resonances-Models and Phenomena, edited by S. Albeverio, L. S. Ferreira, and L. Streit, Lecture Notes in Physics Vol. 211 (Springer-Verlag, Berlin, 1984), p. 27.

[20] B. Simon, Int. J. Quantum Chem. 14, 529 (1978).

[21] D. V. Fedorov, A. S. Jensen, and K. Riisager, Phys. Lett B 312, 1 (1993).

[22] M. V. Zhukov, B. V. Danilin, D. V. Fedorov, J. M. Bang, I. J. Thompson, and J. S. Vaagen, Phys. Rep. 231, 151 (1993).

[23] A. Csótó, Phys. Rev. C 48, 165 (1993).

[24] F. Ajzenberg-Selove, Nucl. Phys. A490, 1 (1988). 


\section{FIGURES}

FIG. 1. Energy eigenvalues of a complex scaled (a) two-body problem with $m_{1}=m_{2}=2$, and $\lambda_{12}=0.6377+i 0.0697 ;(\mathrm{b})-(\mathrm{d})$ three-body problem with $m_{1}=m_{2}=2, m_{3}=4$, $\lambda_{12}=0.6377+i 0.0697$, and $\lambda_{13}=\lambda_{23}=1.0$. The trial wave function is: (b) $\Psi_{1}$, (c) $\Psi_{2}$, and (d) $\Psi_{3}$. The $\theta$ rotation angle is $0.4 \mathrm{rad}$ in each figure.

FIG. 2. Distribution of the continuum energy solutions of the three-body problem specified in Fig. 1, with the trial function $\Psi_{3}$. The solutions are groupped within 0.2 wide energy bins. 
This figure "fig1-1.png" is available in "png" format from: http://arxiv.org/ps/nucl-th/9311022v1 
This figure "fig1-2.png" is available in "png" format from: http://arxiv.org/ps/nucl-th/9311022v1 